\title{
Significance of Pulmonary Rehabilitation in Improving Quality of Life for Subjects With COPD
}

\author{
Jingjing Yang, Ruiyan Lin, Zhenyang $\mathrm{Xu}$, and Hongxia Zhang
}

\begin{abstract}
BACKGROUND: Increasingly, studies have shown that application of pulmonary rehabilitation (PR) may improve the quality of life (QOL) of patients with COPD. However, some studies remain controversial and were limited to small number of participants. We designed a systematic review and meta-analysis to evaluate the efficacy of PR in improving the QOL for subjects with COPD. METHODS: We searched the Cochrane Library, PubMed, EMBASE, and Web of Science up to March 29, 2018, to identify relevant randomized controlled trials that analyzed and evaluated the efficacy of PR in subjects with COPD. Participants were randomly assigned to receive PR (intervention group) or usual care (control group). We used Chronic Respiratory Questionnaire scores, which include 4 important domains (ie, Fatigue, Emotion, Mastery, and Dyspnea) as the evaluating indicators of QOL. Mean differences with $95 \%$ CI were estimated to compare the outcomes of the groups. We also performed subgroup analysis for the pooled results of PR effects in subjects with COPD. In addition, a sensitivity analysis was performed to examine the stability of the combined results. Two reviewers assessed trial quality and extracted data independently. RESULTS: Seventeen randomized controlled trials $(N=1,649$ participants) were identified for the present analysis. In comparing PR groups with usual care groups, we identified significant effects in QOL improvement as measured by the Chronic Respiratory Questionnaire scores for fatigue (Mean difference $0.60,95 \%$ CI $0.36-0.84, P<.001$ ), mastery (Mean difference $0.59,95 \%$ CI $0.32-0.85, P<.001$ ), and dyspnea (Mean difference $0.70,95 \%$ CI $0.46-0.94, P<.001$ ), but no clinically important improvement was found in emotion (Mean difference $0.45,95 \%$ CI $0.23-0.67, P<.001$ ) according to the minimal clinically important difference that we defined as mean difference $\geq 0.5$ units. CONCLUSION: PR may constitute an important component of COPD management and may be beneficial in improving QOL. Key words: pulmonary rehabilitation; COPD; quality of life. [Respir Care 2019;64(1):99-107. ( 2019 Daedalus Enterprises]
\end{abstract}

\section{Introduction}

COPD is one of the most common chronic lung diseases, and it is caused by multiple factors. It is also correlated with

The authors are affiliated with the Department of Respiratory and Critical Care Medicine, Beijing Luhe Hospital, Capital Medical University, Beijing, China.

Supplementary material related to this paper is available at http:// www.rcjournal.com.

Correspondence: Hongxia Zhang, Department of Respiratory and Critical Care Medicine, Beijing Luhe Hospital, Capital Medical University, Xinhuananlu 82, Tongzhou District, Beijing 101100, China. E-mail: hxzhang1026@126.com.

DOI: $10.4187 /$ respcare. 06353 extra-pulmonary effects, including cardiovascular disease, limitation of outdoor activities, and depression. ${ }^{1}$ It has become one of the major causes of morbidity secondary to chronic lung disease worldwide. Despite immense progress made so far, the therapy and management of COPD still lack groundbreaking advancements.

A large number of recent studies focus on pulmonary rehabilitation (PR), which was initially defined in 1974 as a therapy used to reduce COPD symptoms, improve quality of life (QOL), and increase physical and emotional health. According to the American Thoracic Society and the European Respiratory Society, PR is an evidence-based, multidisciplinary, and comprehensive intervention for patients with chronic respiratory diseases. ${ }^{2}$ Since this definition of PR was put forward, it has been recognized as one of the crucial components of the management of 
individuals with chronic respiratory disease. Candemir et $\mathrm{al}^{3}$ evaluated the relationship between the COPD assessment test and improvements after PR and PR efficiency in subjects with COPD. Engel et $\mathrm{al}^{4}$ designed a randomized controlled trial (RCT) to investigate whether the combination of exercise and manual therapy including spinal manipulative therapy produced sustainable improvements in lung function and exercise capacity in 202 subjects with stable mild COPD.

Other studies have explored the effects of PR in subjects with COPD..$^{5-7}$ However, the results remain controversial, and their sample sizes were small. ${ }^{8-11}$ Therefore, the present meta-analysis, based on relevant studies, was performed to comprehensively analyze and evaluate the efficacy of PR in subjects with COPD using Chronic Respiratory Questionnaire (CRQ) scores, which include 4 important domains (ie, Fatigue, Emotion, Mastery, and Dyspnea) to address the aforementioned issue and to draw a convincing conclusion based on a larger sample size. ${ }^{12}$ There were a sufficient number of homogenous studies using the CRQ to support its use to assess the QOL of subjects with COPD. This analysis emphasized that PR might serve as an important component of the management of COPD and might be beneficial in improving QOL.

\section{Methods}

This meta-analysis, which is based on relevant RCTs, was designed to comprehensively analyze and evaluate the efficacy of PR in subjects with COPD. Participants in analyzed studies were randomly assigned to receive PR (intervention group) or usual care (control group). CRQ scores were used, which include 4 important domains for fatigue, emotion, mastery, and dyspnea as the evaluating indicators of QOL.

The inclusion criteria were as follows: (1) the studies were designed as RCTs that compared the effects of PR versus usual care; (2) the studies included participants who had a clinical diagnosis of COPD; (3) the studies included RCTs in which any or all participants were on continuous oxygen; (4) study participants underwent a PR program of any type for $\geq 4$ weeks; and (5) the studies included the CRQ as a primary outcomes. Exclusion criteria were as follows: (1) experimental trials on animals or nonhuman studies; (2) abstracts, letters, editorials, expert opinions, reviews, and case reports; (3) RCTs that focused on participants who were mechanically ventilated; (4) participants who had an exacerbation within 4 weeks before commencement of the intervention; and (5) studies without sufficient data or that did not meet the inclusion criteria.

The Cochrane Library, PubMed, EMBASE, and Web of Science were searched to March 29, 2018. The search terms and procedures were as follows: (1) "chronic ob- structive pulmonary disease"; (2) "rehabilitation" OR "physical training" OR "exercise"; (3) "quality of life" OR "Chronic Respiratory Disease Questionnaire." Searching was filtered by title/abstract with the strategy: (1) AND (2) AND (3). Other related terms, including references of some published studies, were also searched in English. Two assessors independently screened the titles and abstracts of each study. Once relevant studies were selected, the full texts were obtained for further evaluation.

Two reviewers assessed the quality of all of the included studies. The total scores of each study are presented in Table 1. The quality assessment of RCTs was evaluated and scored using the previously validated 5-point Jadad scale. ${ }^{13}$ Further details of this scale are given in the notes of Table 1 . Studies with scores of $\geq 3$ were considered of good quality. In addition, the risk of bias for each RCT and the risk of bias across all RCTs were evaluated and shown with figures generated using RevMan 5.2 software. ${ }^{14}$

Data for the analysis of the efficacy of PR intervention versus usual care in subjects with COPD were extracted independently by two reviewers, and disagreement was resolved through discussion. The extracted contents, including study demographics, publication years, trial design, rehabilitation interventions, main inclusion criteria, duration of rehabilitation intervention, outcomes, and Jadad score of each study, were displayed using a standardized form. Data collected were entered into RevMan 5.2 software for analysis. ${ }^{14}$

The data of comparable outcomes between the intervention and control groups were analyzed using the standard statistical procedures provided in RevMan 5.2 (Table 1). ${ }^{14}$ The mean difference of the change in $\mathrm{CRQ}^{13,15}$ and the associated $95 \%$ CI range were also measured. For the change in CRQ, higher scales were considered better and a change of 0.5 units was considered to be a clinically important difference. The heterogeneity between studies was evaluated using the chi-square-based Q statistical test, ${ }^{16}$ with $P$ value and $I^{2}$ statistic, ranging from $0 \%$ to $100 \%$, to quantify the effect of heterogeneity. A $P$ value $\leq .10$ was deemed to represent significant heterogeneity. ${ }^{17}$ Pooled mean difference was estimated using a randomeffects model (the DerSimonian and Laird method ${ }^{18}$ ). On the contrary, if statistical heterogeneity was not observed $(P>.10)$, a fixed-effects model (the Mantel-Haenszel method $^{19}$ ) was used. The effects of outcome measures were considered to be statistically significant if pooled mean differences with $95 \% \mathrm{CI}$ did not overlap with 0 . A subgroup analysis was performed for the pooled results of the effect of PR in subjects with COPD according to the Jadad score. In addition, a sensitivity analysis was performed to examine the stability of the combined results. 
Pulmonary Rehab AND ImPRoved QOL IN COPD

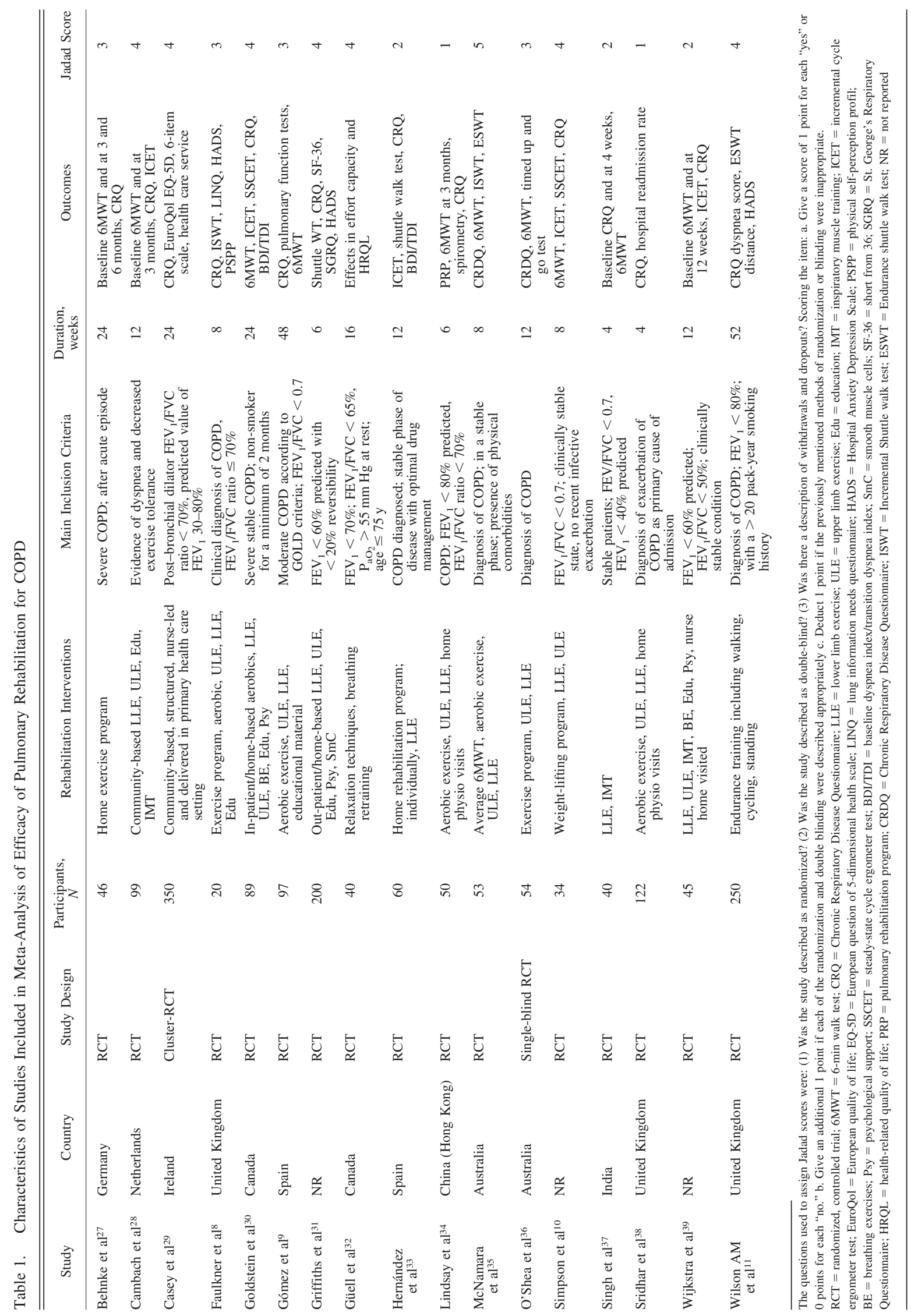




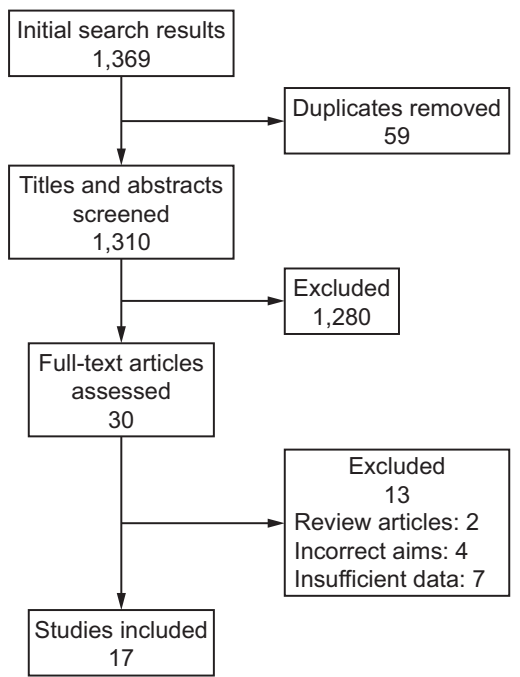

Fig. 1. Flow chart.

\section{Results}

The database search yielded a total of 1,369 records of citations; there were 1,310 after duplicates were removed. By screening the titles and abstracts of the 1,310 citations, 1,280 studies were excluded preliminarily, we obtained full texts for 30 studies for further evaluation. After reading the full texts of the 30 studies, 13 studies were excluded further ( 2 studies were review articles, ${ }^{20,21} 4$ articles had different objectives, ${ }^{3,5,7,22}$ and 7 studies lacked adequate data 4,6,23-26). Eventually, 17 studies ${ }^{8-11,27-39}$ ( $N=1,649$ participants) were included in this metaanalysis. The sample sizes ranged from 20 to 350 subjects (Table 1). Two studies had a Jadad score of 1 , 3 studies had a Jadad score of 2, 4 studies had a Jadad score of 3, 7 studies had a Jadad score of 4, and 1 study had a Jadad score of 5. According to the definition of good quality, approximately $71 \%$ of studies exhibited good quality.

The detailed search process and summary of studies are shown in Figure 1. The other characteristics of each study are shown in Table 1.

Graphs were generated to further identify the risk of bias of the included studies. The risk of bias for each RCT was presented as percentages across all included studies, and the risk of bias for each included study was displayed (see the supplementary materials at http://www.rcjournal. com). The risk-of-bias graphs indicated generally good methodological quality. Blinding issues were a low risk of bias in these studies. A high risk of bias was mainly observed relevant to subject selection issues, and an unclear risk of bias was observed in selection and detection biases. A low risk of bias was observed in other biases.

\section{Efficacy of Rehabilitation in Improving CRQ Scale}

As shown in Figure 2, a significant result was obtained with a pooled mean difference of 0.60 (95\% CI 0.36-0.84, $P<.001)$ comparing the effects of PR versus usual care in improving the QOL of subjects with COPD according to the change in CRQ (Fatigue). The pooled analysis was estimated using random-effects models because significant heterogeneity among studies was found $(P<.001$ and $I^{2}=65 \%$ ). In addition, studies with a high risk of detection and attrition bias were omitted, and the remaining studies were combined to further evaluate the effect of PR. When the studies with a high detection bias were omitted, ${ }^{8,28,39}$ the significant combined results were also observed with a pooled mean difference of 0.57 (95\% CI $0.31-0.83, P<.001)$. Similarly, when the studies with a high attrition bias were omitted, $8,9,27,29,33,34$ the pooled results had a mean difference of 0.66 (95\% CI 0.43-0.89, $P<.001)$.

This study also analyzed the efficacy of PR in improving the QOL of subjects with COPD according to the change in CRQ (Emotion). However, a combined analysis result of 17 studies showed that PR had no significant effect in improving the CRQ (Emotion) scale. No significant change in CRQ (Emotion) was observed in subjects with COPD receiving PR in comparison with subjects receiving usual care, with a pooled mean difference of 0.45 (95\% CI $0.23-0.67, P<.001$ ) (using the random-effects model) according to the minimal clinically important difference of 0.5 units (Fig. 3). Meanwhile, the efficacy of PR was further evaluated by omitting studies with a high risk of detection and attrition bias, then combining the remaining studies. When the studies with a high detection bias $8,28,39$ and a high attrition bias $8,9,24,29,33,34$ were omitted, the changes did not achieve clinical importance according to the minimal clinically important difference of 0.5 units, although statistically significant results were observed with pooled mean differences of $0.45(95 \%$ CI $0.20-0.70, P<.001)$ (using the random-effects model) and 0.46 (95\% CI 0.29-0.64, $P<.001$ ) (using the fixed-effects model).

A clinically important change in CRQ (Mastery) was observed in subjects receiving PR compared with patients receiving usual care, with a pooled mean difference of 0.59 (95\% CI 0.32-0.85, $P<.001$ ). Meanwhile, the efficacy of PR was further evaluated by omitting studies with a high risk of detection bias and attrition bias and combining the remaining studies. After studies with a high detection $\operatorname{bias}^{8,28,39}$ and a high attrition $\operatorname{bias}^{8,9,29,33,34}$ were omitted, the combined results also showed clinically important changes in the intervention groups, with the pooled mean differences being 0.53 (95\% CI 0.24-0.82, $P=<.001)$ and 0.58 (95\% CI $0.32-0.85, P<.001)$, respectively. 
Pulmonary Rehab and Improved QOL in COPD

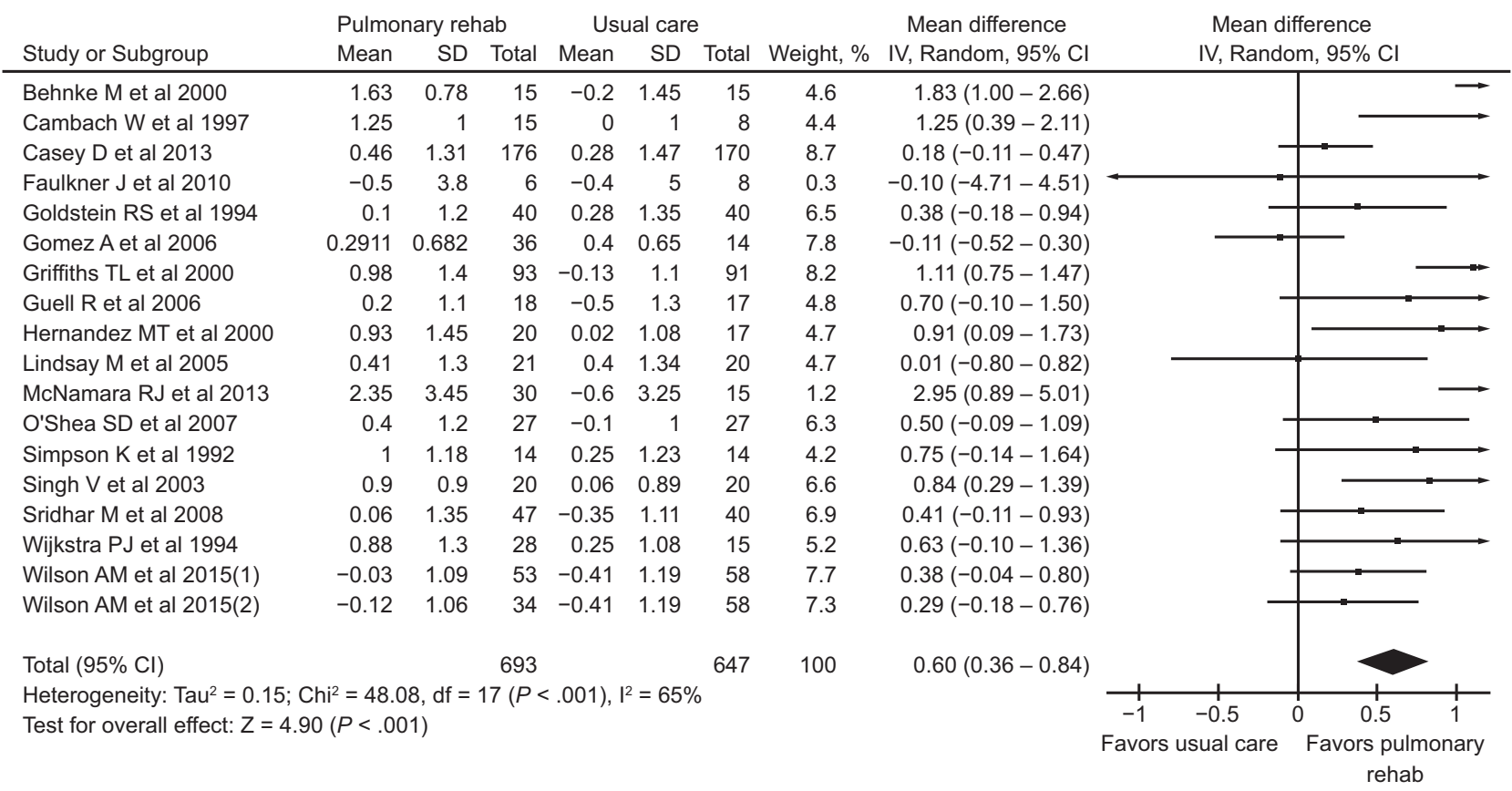

Fig. 2. Forest plot of the efficacy of pulmonary rehabilitation in improving the quality of life of subjects with COPD according to change in Chronic Respiratory Disease Questionnaire (Fatigue).

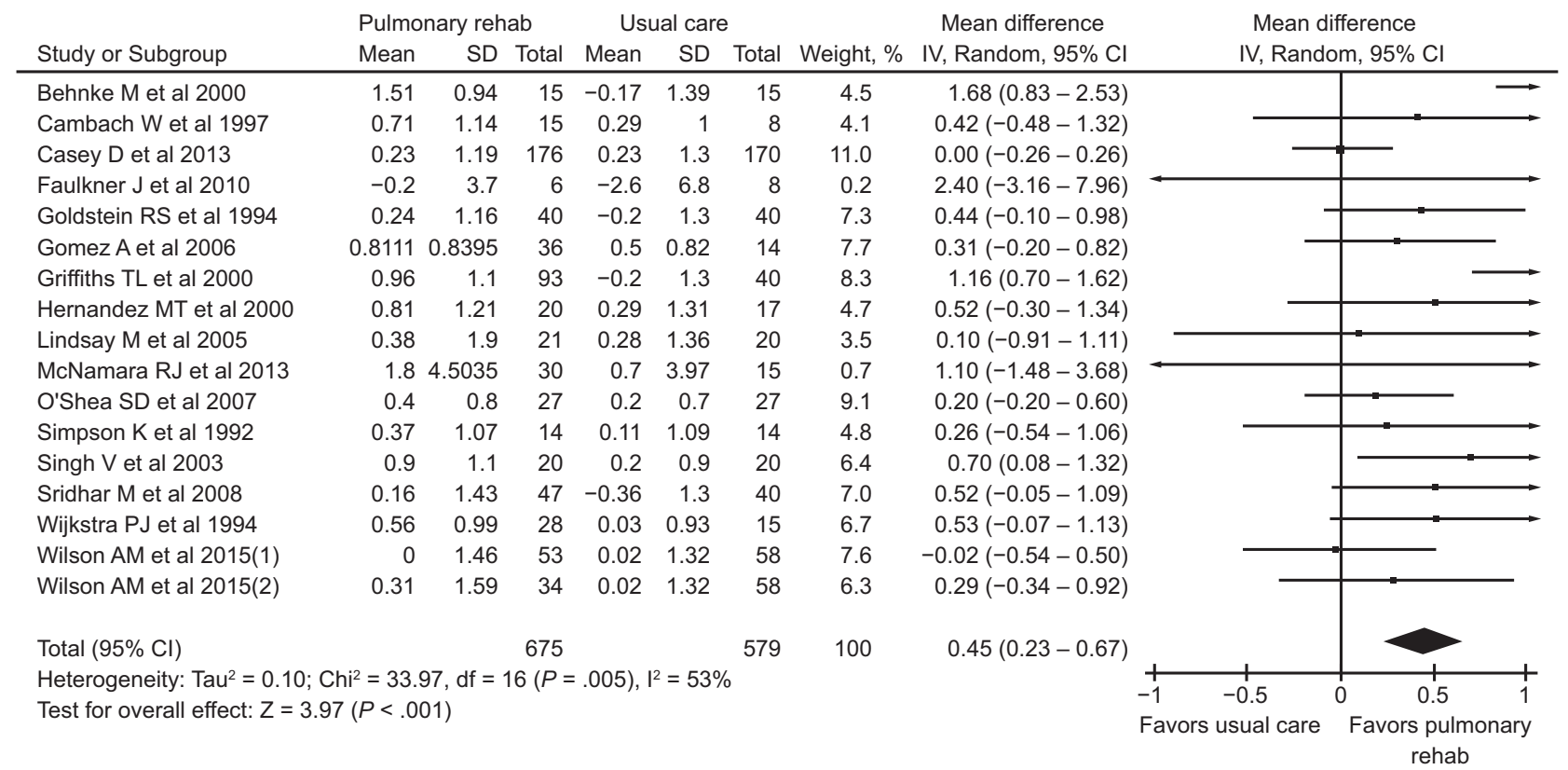

Fig. 3. Forest plot of the efficacy of pulmonary rehabilitation in improving the quality of life of subjects with COPD according to change in Chronic Respiratory Disease Questionnaire (Emotion).

Considering the significant heterogeneity, random-effects models were used to evaluate the effect (Fig. 4).

A clinically important change in CRQ (Mastery) was also found in the PR groups, with a pooled mean difference of 0.70 (95\% CI 0.46-0.94, $P<.001$ ). After studies with a high detection bias $^{8,28,39}$ and a high attrition bias $8,9,24,29,33,34$ were omitted, the combined results also showed clinically important changes in the intervention groups, with pooled mean differences of 0.67 (95\% CI $0.41-0.94, P<.001$ ) and 0.74 (95\% CI $0.45-1.02, P<.001$ ), respectively. Because of the significant heterogeneity, random-effect models were used to evaluate the effect (Fig. 5). 
Pulmonary Rehab ANd Improved QOL in COPD

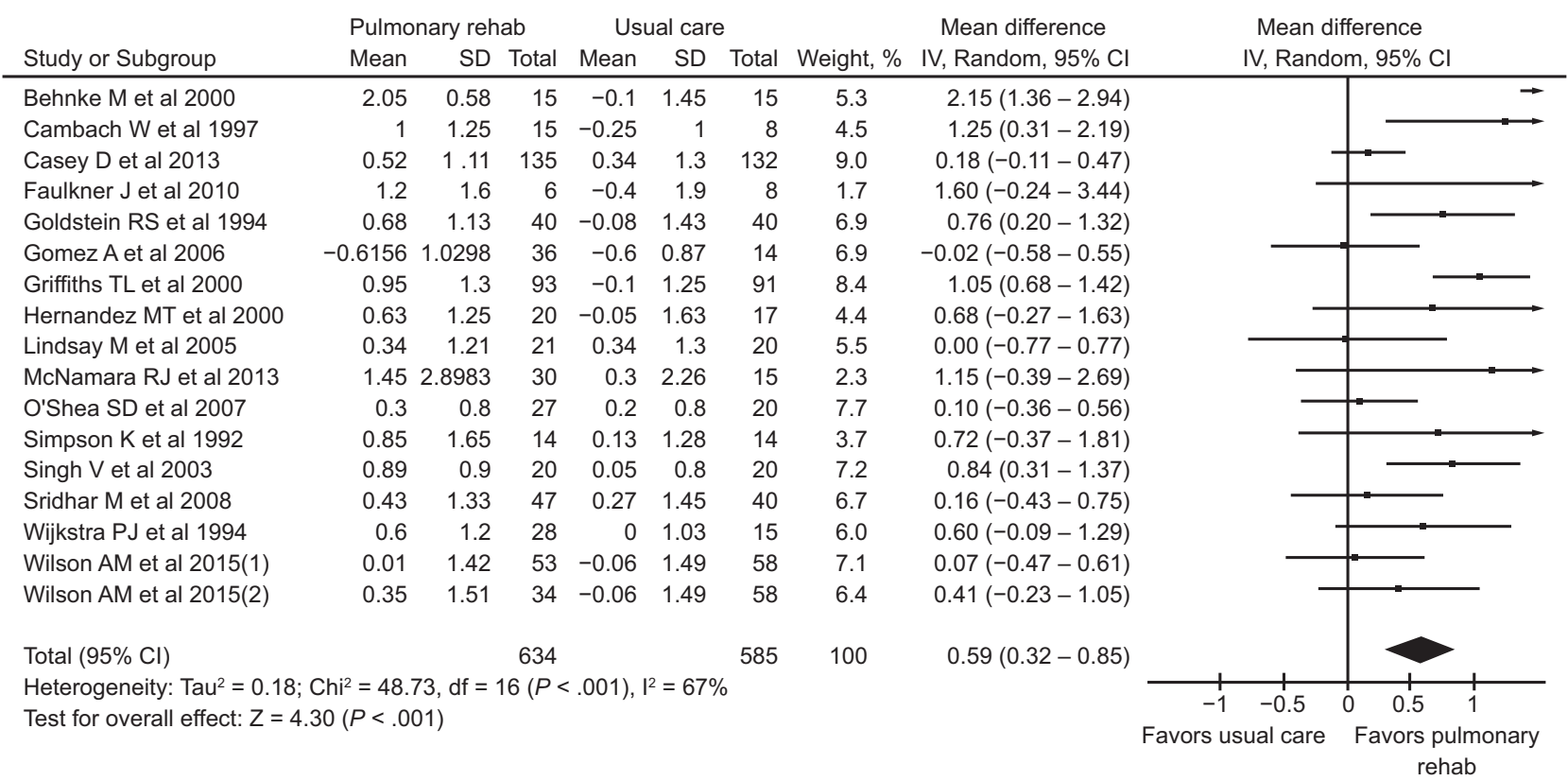

Fig. 4. Forest plot of the efficacy of pulmonary rehabilitation in improving the quality of life of subjects with COPD according to change in Chronic Respiratory Disease Questionnaire (Mastery).

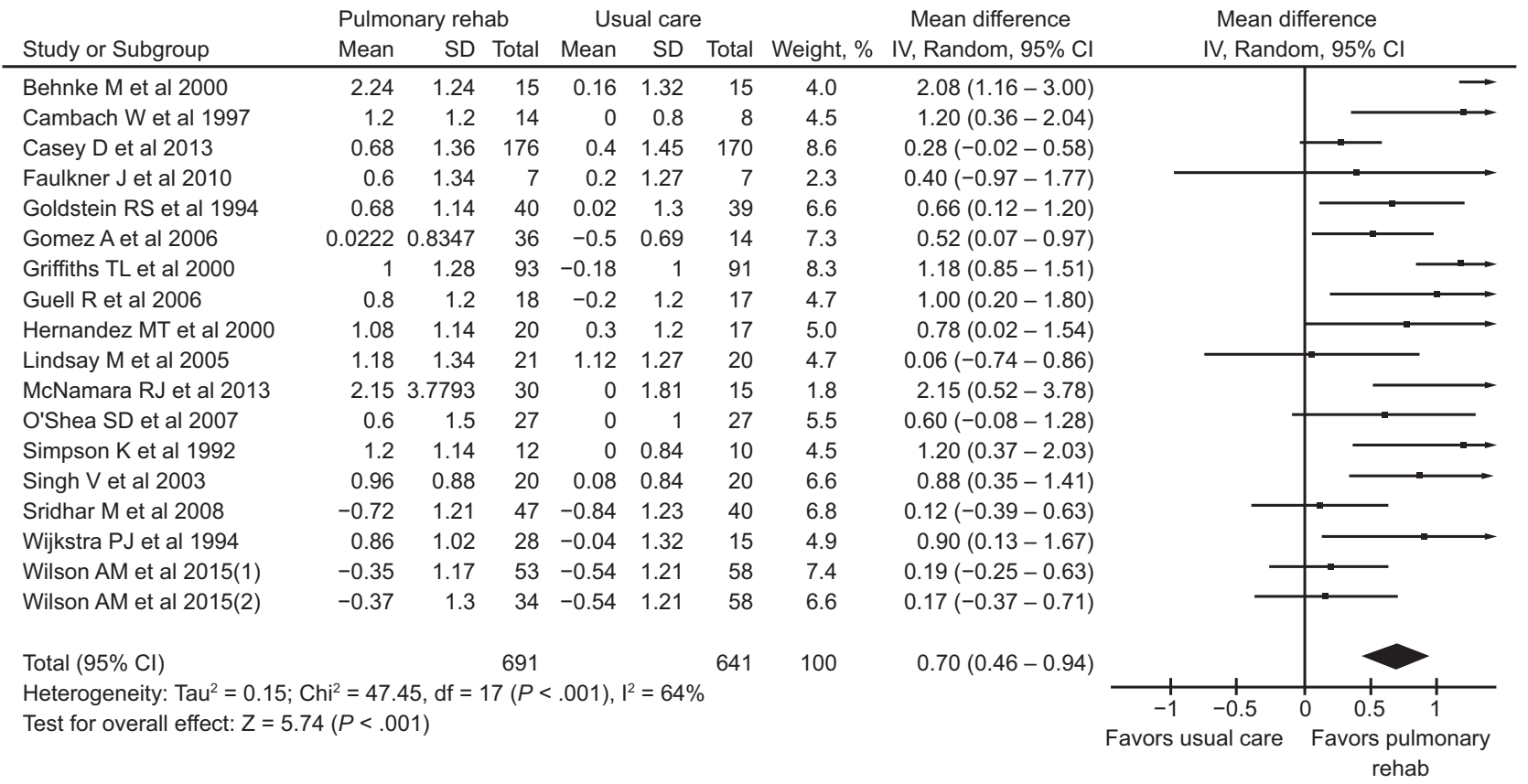

Fig. 5. Forest plot of the efficacy of pulmonary rehabilitation in improving the quality of life of subjects with COPD according to change in Chronic Respiratory Disease Questionnaire (Dyspnea).

\section{Subgroup and Sensitivity Analyses}

A subgroup analysis was performed for the changes in mean difference in improving the CRQ scale according to Jadad scores to further explore the efficacy of PR in subjects with COPD (Table 2). For studies experiencing higher scores of 3-4, statistically significant effects of PR were observed in the CRQ scales of Fatigue (Mean difference $0.47,95 \%$ CI $0.33-0.62, P<.001$ ), Emotion (Mean difference $0.31,95 \%$ CI $0.16-0.46, P<.001)$, Mastery (Mean difference $0.50,95 \% \mathrm{CI} 0.34-0.66, P<.001$ ), and Dyspnea (Mean difference 0.64, 95\% CI 0.49-0.79, 
Pulmonary Rehab AND IMPROVED QOL In COPD

Table 2. Pooled Results of Analysis by Jadad Scores for the Efficacy of Pulmonary Rehabilitation for COPD

\begin{tabular}{|c|c|c|c|c|c|}
\hline \multirow{2}{*}{ Subgroups } & \multicolumn{2}{|c|}{ Pooled Results } & \multicolumn{3}{|c|}{ Heterogeneity } \\
\hline & Mean Difference $(95 \% \mathrm{CI})$ & $P$ & $I^{2}$ & $P$ & Analytical Effect Model \\
\hline \multicolumn{6}{|l|}{ Jadad score of $1-2$} \\
\hline CRQ (Fatigue) & $0.57(0.28-0.86)$ & .001 & $0 \%$ & .43 & Fixed-effect model \\
\hline CRQ (Emotion) & $0.53(0.22-0.83)$ & $<.001$ & $0 \%$ & .91 & Fixed-effect model \\
\hline CRQ (Mastery) & $0.48(0.19-0.78)$ & .001 & $15 \%$ & .32 & Fixed-effect model \\
\hline CRQ (Dyspnea) & $0.53(0.25-0.82)$ & $<.001$ & $41 \%$ & .15 & Fixed-effect model \\
\hline \multicolumn{6}{|l|}{ Jadad score of 3-4 } \\
\hline CRQ (Fatigue) & $0.47(0.33-0.62)$ & $<.001$ & $71 \%$ & $<.001$ & Random-effect model \\
\hline CRQ (Emotion) & $0.31(0.16-0.46)$ & $<.001$ & $68 \%$ & $<.001$ & Random-effect model \\
\hline CRQ (Mastery) & $0.50(0.34-0.66)$ & $<.001$ & $77 \%$ & $<.001$ & Random-effect model \\
\hline CRQ (Dyspnea) & $0.64(0.49-0.79)$ & $<.001$ & $70 \%$ & $<.001$ & Random-effect model \\
\hline \multicolumn{6}{|l|}{ Jadad score of 5} \\
\hline CRQ (Fatigue) & $2.95(0.89-5.01)$ & $<.001$ & - & - & - \\
\hline CRQ (Emotion) & $1.10(-1.48-3.68)$ & .40 & - & - & - \\
\hline CRQ (Mastery) & $1.15(-0.39-2.69)$ & .14 & - & - & - \\
\hline CRQ (Dyspnea) & $2.15(0.52-3.78)$ & .01 & - & - & - \\
\hline
\end{tabular}

$P<.001)$, although a significant heterogeneity was found. However, only the changes in Mastery and Dyspnea achieved clinical importance according to the minimal clinically important difference of 0.5 units. For studies with lower scores of $1-2$, statistically significant effects of PR were similarly observed in the CRQ scales of Fatigue (Mean difference $0.57,95 \%$ CI $0.28-0.86, P<.001$ ), Emotion (Mean difference $0.53,95 \%$ CI $0.22-0.83, P<.001$ ), and Dyspnea (Mean difference $0.53,95 \%$ CI $0.25-0.82, P<$ .001 ), but not in Mastery (Mean difference $0.48,95 \%$ CI $0.19-0.78, P=.001)$, according to the minimal clinically important difference of 0.5 units, with good homogeneity achieved using fixed-effects models. For the one study with high methodological quality (Jadad score $=5$ ), an extremely significant clinical difference was observed in the changes in the CRQ scales of Fatigue (Mean difference 2.95 , 95\% CI $0.89-5.01, P<.001$ ) and Dyspnea (Mean difference 2.15, 95\% CI 0.52-3.78, $P=.01$ ), but no significant difference was found in the CRQ scales of Emotion (Mean difference $1.10,95 \%$ CI -1.48 to 3.68 , $P=.40$ ) and Mastery (Mean difference $1.15,95 \% \mathrm{CI}-0.39$ to $2.69, P=.14$ ).

A sensitivity analysis was conducted to examine the stability of the combined results of the efficacy of PR in improving QOL, and to identify the source of heterogeneity by omitting a single study. The sensitivity analysis of the CRQ scale of Fatigue showed that the combined results experienced high stability by omitting each study, and no particular study significantly affected the pooled results, with the combined mean differences ranging from 0.53 (95\% CI $0.31-0.76$ ) after omitting the first study to 0.63 (95\% CI 0.37-0.89) after omitting the last study. Meanwhile, similar sensitivity analysis results were also obtained on the CRQ scales of Dyspnea, Emotion, and Mastery.

\section{Discussion}

COPD has become one of the major causes of morbidity in people with chronic lung diseases worldwide as a result of its increasing prevalence due to multiple factors such as nicotine addiction and deteriorating air quality. Based on the 2017 report of the Global Initiative for Chronic Obstructive Lung Disease, which focused primarily on the revised and novel parts of the Global Strategy for the Diagnosis, Management, and Prevention of COPD, PR is considered one of the most effective therapies for improving physical performance and QOL by reducing dyspnea. ${ }^{40}$ Furthermore, PR has shown physiological, symptom-reducing, psychosocial, and health economic benefits for patients with chronic respiratory diseases. Still, it is underutilized worldwide. ${ }^{41}$ A review by Gloeckl et al ${ }^{20}$ showed that counseling about available social services is particularly crucial for older subjects when providing PR. These available social services include possible home care or services for severe disability and preparation of therapeutic appliances. For patients receiving long-term oxygen treatment, accrediting severely disabled persons to use local public transport can provide practical relief and relieve the financial burden. ${ }^{20}$

This systematic review summarized 17 RCTs involving 1,649 participants with COPD. In these studies, subjects received PR for at least 4 weeks and at most 52 weeks. ${ }^{9} 11,37,38$ This meta-analysis demonstrated the efficacy of PR in improving the QOL of subjects with COPD. The results statistically support the conclusion that the 
CRQ scale was significantly improved in subjects receiving PR compared with those receiving usual care, with clinically important mean differences in changes for Fatigue (Mean difference 0.60, 95\% CI 0.36-0.84, $P<.001$ ), Mastery (Mean difference 0.59, 95\% CI 0.32-0.85, $P<.001$ ), and Dyspnea (Mean difference $0.70,95 \%$ CI $0.46-0.94, P<.001)$. However, no clinically important improvement was found in changes for Emotion (Mean difference $0.45,95 \%$ CI $0.23-0.67, P<.001$ ) according to the minimal clinically important difference of 0.5 units, despite statistically significant results. These results were different from the findings of McCarthy et al, ${ }^{21}$ who found that PR could improve all aspects of the CRQ scale, including Emotion (Mean difference 0.56, 95\% CI 0.340.78); however, according to the criteria, that result was supported by low-quality evidence. In our review, the studies with high risk of detection and attrition bias were omitted and the remaining studies were combined to further evaluate the effects of PR. Significant results were obtained with pooled mean differences of 0.57 (95\% CI 0.31$0.83, P<.001)$ and 0.66 (95\% CI $0.43-0.89, P<.001$ ), respectively, implying high-quality and stable evidence for Fatigue. Meanwhile, similar results were also achieved for Mastery ([Mean difference 0.53, 95\% CI 0.24-0.82, $P<.001$ ] and [Mean difference $0.58,95 \%$ CI $0.32-0.85$, $P<.001]$ ) and Dyspnea ([Mean difference 0.67, 95\% CI $0.41-0.94, P<.001]$ and [MD 0.74, 95\% CI 0.45-1.02, $P<.001])$. Apart from this, the significance of results did not undergo any changes, meeting the minimal clinically important difference of 0.5 units on further evaluation of the effects of PR on Emotion.

This meta-analysis had several limitations. The main limitation was the inconsistency in PR programs and the duration of rehabilitation intervention. Treatment duration ranged from 4-52 weeks. ${ }^{9,37,38}$ In the study by Wilson et al, ${ }^{11}$ participants received the longest rehabilitation intervention lasting $1 \mathrm{y}$. Their results showed no statistically significant difference in the change in CRQ (Dyspnea) score (primary end point) or other domains of CRQ after 12 months, which amounted to 0.19 ( -0.26 to 0.64$)$ units, between the groups. Their findings indicated a negative effect of the program and appealed for other methods of sustaining the benefits of PR. The second limitation of this meta-analysis was the sample sizes of the included studies, which ranged from 20 to 350 subjects. Considering the natural conditions of the trials, every study meeting the inclusion criteria was retained. Fortunately, only $2-17$ studies had a small sample size $<40$ participants. ${ }^{8}{ }^{80}$ Finally, the effects of PR were also influenced by additional factors, such as age of participants, smoking history, previous respiratory tract infection and infection time, and severe or uncontrolled comorbid disease, which should also be taken into consideration.
The search strategy for this review was comprehensive, broad, and systematic, with hand-searching of some references of included studies and previous systematic reviews. Keeping in mind considerable heterogeneity in the study design of the included trials, especially in the PR interventions, more studies should be designed to focus on comparing the effects of different PR interventions along with usual care. In addition, considering many studies had few subjects, further studies with sufficient sample size should be performed to provide more clinically convincing results for the efficacy of PR.

\section{Conclusion}

This analysis showed that PR could significantly improve the QOL of subjects with COPD in terms of Fatigue, Mastery, and Dyspnea compared with usual care. In terms of Emotion, the results were statistically significant and indicated potential clinical effectiveness, although they did not meet the criteria of the minimal clinically important difference of 0.5 units. In conclusion, despite many limitations and influencing factors, the analysis indicated that PR might improve the QOL of patients with COPD.

\section{REFERENCES}

1. Köktürk N, Gürgün A, Şen E, Kocabaş A, Polatlı M, Naycı SA, et al. The view of the Turkish Thoracic Society on the report of the GOLD 2017 Global Strategy for the Diagnosis, Management, and Prevention of COPD. Turk Thorac J 2017;18(2):57-64.

2. Spruit MA, Singh SJ, Garvey C, ZuWallack R, Nici L, Rochester C, et al. An official American Thoracic Society/European Respiratory Society statement: key concepts and advances in pulmonary rehabilitation. Am J Respir Crit Care Med 2013;188(8):e13-e64.

3. Candemir I, Kaymaz D, Ergun P, Demir N, Egesel N, Sengul F. Assessment of pulmonary rehabilitation efficacy in chronic obstructive pulmonary disease patients using the chronic obstructive pulmonary disease assessment test. Expert Rev Respir Med 2015;9(4): 487-492.

4. Engel RM, Wearing J, Gonski P, Vemulpad S. The effect of combining manual therapy with exercise for mild chronic obstructive pulmonary disease: study protocol for a randomised controlled trial. Trials 2017;18(1):282.

5. Pradella CO, Belmonte GM, Maia MN, Delgado CS, Luise AP, Nascimento OA, et al. Home-based pulmonary rehabilitation for subjects with COPD: a randomized study. Respir Care 2015;60(4): 526-532.

6. Prunera-Pardell MJ, Padín-López S, Domenech-Del Rio A, GodoyRamírez A. Effectiveness of a respiratory rehabilitation programme in patients with chronic obstructive pulmonary disease. Enferm Clin 2018;28(1):5-12.

7. Sandoz JS, Roberts MM, Cho JG, Wheatley JR. Magnitude of exercise capacity and quality of life improvement following repeat pulmonary rehabilitation in patients with COPD. Int J Chron Obstruct Pulmon Dis 2017;12:1085-1091.

8. Faulkner J, Walshaw E, Campbell J, Jones R, Taylor R, Price D, et al. The feasibility of recruiting patients with early COPD to a pilot trial assessing the effects of a physical activity intervention. Prim Care Respir J 2010;19(2):124-130. 
9. Gómez A, Román M, Larraz C, Esteva M, Mir I, Thomás V, et al. [Efficacy of respiratory rehabilitation on patients with moderate COPD in primary care and maintenance of benefits at 2 years]. Aten Primaria 2006;38(4):230-233.

10. Simpson K, Killian K, McCartney N, Stubbing DG, Jones NL. Randomised controlled trial of weightlifting exercise in patients with chronic airflow limitation. Thorax 1992;47(2):70-75.

11. Wilson AM, Browne P, Olive S, Clark A, Galey P, Dix E, et al. The effects of maintenance schedules following pulmonary rehabilitation in patients with chronic obstructive pulmonary disease: a randomised controlled trial. BMJ Open 2015;5(3):e005921.

12. Reda AA, Kotz D, Kocks JW, Wesseling G, van Schayck CP. Reliability and validity of the clinical COPD questionnaire and chronic respiratory questionnaire. Respir Med 2010;104(11):1675-1682.

13. Clark HD, Wells GA, Huët C, McAlister FA, Salmi LR, Fergusson $\mathrm{D}$, et al. Assessing the quality of randomized trials: reliability of the Jadad scale. Control Clin Trials 1999;20(5):448-452.

14. Review Manager (RevMan), version 5.2. Copenhagen: The Nordic Cochrane Centre, The Cochrane Collaboration, 2012.

15. Wijkstra PJ, TenVergert EM, Van Altena R, Otten V, Postma DS, Kraan J, et al. Reliability and validity of the chronic respiratory questionnaire (CRQ). Thorax 1994;49:465e7.

16. Lau J, Ioannidis JP, Schmid CH. Quantitative synthesis in systematic reviews. Ann Intern Med 1997;127(9):820-826.

17. University of York Centre for Reviews and Dissemination. Systematic Reviews: CRD's Guidance for Undertaking Reviews in Health Care. York: CRD, University of York, 2009.

18. DerSimonian R, Laird N. Meta-analysis in clinical trials. Control Clin Trials 1986;7(3):177-188.

19. Mantel N, Haenszel W. Statistical aspects of the analysis of data from retrospective studies of disease. J Natl Cancer Inst 1959;22(4): 719-748.

20. Gloeckl R, Schneeberger T, Jarosch I, Kenn K. Pulmonary rehabilitation and exercise training in chronic obstructive pulmonary disease. Dtsch Arztebl Int 2018;115(8):117-123.

21. McCarthy B, Casey D, Devane D, Murphy K, Murphy E, Lacasse Y. Pulmonary rehabilitation for chronic obstructive pulmonary disease. Cochrane Database Syst Rev 2015;(2):CD003793.

22. Chen YW, Camp PG, Coxson HO, Road JD, Guenette JA, Hunt MA, et al. A comparison of pain, fatigue, dyspnea and their impact on quality of life in pulmonary rehabilitation participants with chronic obstructive pulmonary disease. COPD 2018;15(1):65-72.

23. Güell R, Casan P, Belda J, Sangenis M, Morante F, Guyatt GH, et al. Long-term effects of outpatient rehabilitation of COPD: a randomized trial. Chest 2000;117(4):976-983.

24. Porto EF, Castro AAM, Cortopassi F, de Souza GF, Jardim JR Variability in quality of life outcomes following a pulmonary rehabilitation program in patients with COPD. J Cardiopulm Rehabil Prev 2018;38(2):118-123.

25. Troosters T, Gosselink R, Decramer M. Short- and long-term effects of outpatient rehabilitation in patients with chronic obstructive pulmonary disease: a randomized trial. Am J Med 2000;109(3):207212

26. Wu M, Zhou LQ, Li S, Zhao S, Fan HJ, Sun JM, et al. Efficacy of patients' preferred exercise modalities in chronic obstructive pulmonary disease: a parallel-group, randomized, clinical trial. Clin Respir J 2017;12(4):1581-1590.

27. Behnke M, Taube C, Kirsten D, Lehnigk B, Jörres RA, Magnussen $\mathrm{H}$. Home-based exercise is capable of preserving hospital-based im- provements in severe chronic obstructive pulmonary disease. Respir Med 2000;94(12):1184-1191.

28. Cambach W, Chadwick-Straver RV, Wagenaar RC, van Keimpema AR, Kemper HC. The effects of a community-based pulmonary rehabilitation programme on exercise tolerance and quality of life: a randomized controlled trial. Eur Respir J 1997;10(1):104-113.

29. Casey D, Murphy K, Devane D, Cooney A, McCarthy B, Mee L, Newell $\mathbf{J}$, et al. The effectiveness of a structured education pulmonary rehabilitation programme for improving the health status of people with moderate and severe chronic obstructive pulmonary disease in primary care: the PRINCE cluster randomised trial. Thorax 2013;68(10):922-928.

30. Goldstein RS, Gort EH, Stubbing D, Avendano MA, Guyatt GH. Randomised controlled trial of respiratory rehabilitation. Lancet 1994; 344(8934):1394-1397.

31. Griffiths TL, Burr ML, Campbell IA, Lewis-Jenkins V, Mullins J, Shiels K, et al. Results at 1 year of outpatient multidisciplinary pulmonary rehabilitation: a randomised controlled trial. Lancet 2000; 355(9201):362-368.

32. Güell R, Resqueti V, Sangenis M, Morante F, Martorell B, Casan P, et al. Impact of pulmonary rehabilitation on psychosocial morbidity in patients with severe COPD. Chest 2006;129(4):899-904.

33. Hernández MT, Rubio TM, Ruiz FO, Riera HS, Gil RS, Gómez JC. Results of a home-based training program for patients with COPD. Chest 2000;118(1):106-114.

34. Lindsay M, Lee A, Chan K, Poon P, Han LK, Wong WC, et al. Does pulmonary rehabilitation give additional benefit over tiotropium therapy in primary care management of chronic obstructive pulmonary disease? Randomized controlled clinical trial in Hong Kong Chinese. J Clin Pharm Ther 2005;30(6):567-573.

35. McNamara RJ, McKeough ZJ, McKenzie DK, Alison JA. Waterbased exercise in COPD with physical comorbidities: a randomised controlled trial. Eur Respir J 2013;41(6):1284-1291.

36. O'Shea SD, Taylor NF, Paratz JD. A predominantly home-based progressive resistance exercise program increases knee extensor strength in the short-term in people with chronic obstructive pulmonary disease: a randomised controlled trial. Aust J Physiother 2007; 53(4):229-237

37. Singh V, Khandelwal DC, Khandelwal R, Abusaria S. Pulmonary rehabilitation in patients with chronic obstructive pulmonary disease. Indian J Chest Dis Allied Sci 2003;45(1):13-17.

38. Sridhar M, Taylor R, Dawson S, Roberts NJ, Partridge MR. A nurse led intermediate care package in patients who have been hospitalised with an acute exacerbation of chronic obstructive pulmonary disease. Thorax 2008;63(3):194-200.

39. Wijkstra PJ, Van Altena R, Kraan J, Otten V, Postma DS, Koëter $\mathrm{GH}$. Quality of life in patients with chronic obstructive pulmonary disease improves after rehabilitation at home. Eur Respir J 1994; 7(2):269-273.

40. Vogelmeier CF, Criner GJ, Martínez FJ, Anzueto A, Barnes PJ, Bourbeau J, et al. Global Strategy for the Diagnosis, Management and Prevention of Chronic Obstructive Lung Disease 2017 Report: GOLD Executive Summary. Respirology 2017;22(3):575-601.

41. Rochester CL, Vogiatzis I, Holland AE, Lareau SC, Marciniuk DD, Puhan MA, et al. An official American Thoracic Society/European Respiratory Society policy statement: enhancing implementation, use, and delivery of pulmonary rehabilitation. Am J Respir Crit Care Med 2015;192(11):1373-1386. 